\title{
Dynamics of microRNAs in bull spermatozoa
}

\author{
Aruna Govindaraju', Alper Uzun², LaShonda Robertson', Mehmet O Atli ${ }^{3,4}$, Abdullah Kaya ${ }^{5}$, Einko Topper ${ }^{5}$, \\ Elizabeth A Crate ${ }^{1,6}$, James Padbury ${ }^{2}$, Andy Perkins ${ }^{7}$ and Erdogan Memili ${ }^{1 *}$
}

\begin{abstract}
Background: MicroRNAs are small non-coding RNAs that regulate gene expression and thus play important roles in mammalian development. However, the comprehensive lists of microRNAs, as well as, molecular mechanisms by which microRNAs regulate gene expression during gamete and embryo development are poorly defined. The objectives of this study were to determine microRNAs in bull sperm and predict their functions.

Methods: To accomplish our objectives we isolated miRNAs from sperm of high and low fertility bulls, conducted microRNA microarray experiments and validated expression of a panel of microRNAs using real time RT-PCR. Bioinformatic approaches were carried out to identify regulated targets.

Results: We demonstrated that an abundance of microRNAs were present in bovine spermatozoa, however, only seven were differentially expressed; hsa-aga-3155, -8197, -6727, -11796, -14189, -6125, -13659. The abundance of miRNAs in the spermatozoa and the differential expression in sperm from high vs. low fertility bulls suggests that the miRNAs possibly play important functions in the regulating mechanisms of bovine spermatozoa.
\end{abstract}

Conclusion: Identification of specific microRNAs expressed in spermatozoa of bulls with different fertility phenotypes will help better understand mammalian gametogenesis and early development.

Keywords: Uncompensatory fertility, Mammalian, Fertilization, Embryonic development

\section{Background}

Fertilization is the most important factor controlling mammalian reproduction in which a developmentally competent spermatozoon fertilizes the egg and sets the stage for egg activation and embryo development. Even though producing an abundant amount of spermatozoa with normal motility and morphology, the fertility of some bulls is low which may be due to molecular defects in the sperm [1]. Molecular and cellular integrity of sperm is not only important for fertilization but also for embryo development and later fetal development. MicroRNAs are small non-coding RNAs that regulate gene expression of messenger RNA which are abundant in bull spermatozoa [2]. We asked: What are the microRNAs (miRNAs) that are present in the mature male gamete? What does their presence in the spermatozoa mean? What roles do these miRNAs play in fertilization, egg activation, early and later development? Answers to these fundamental questions remain unknown at the present.

\footnotetext{
* Correspondence: em149@ads.msstate.edu

${ }^{1}$ Departments of Animal and Dairy Sciences, Mississippi State University, Mississippi State, MS 39762, USA

Full list of author information is available at the end of the article
}

Successful development is a highly choreographed process with precision in the timing and degree of gene expression at each step. The control of gene expression is exhibited at each point of the pathway from transcription to translation. MiRNAs are among the newly recognized factors associated with important post-transcriptional regulation of gene expression. As such, miRNAs have gained special interest and their roles during various developmental stages are the subject of many investigations. MiRNAs are tissue specific and they are involved in specific cell differentiation processes [3]. Discovery of candidate miRNAs will allow understanding of the regulatory pathway(s) of particular genes. Thereby, the spatial and temporal expression and even the function of individual genes can be defined.

MiRNAs are generated from double-stranded RNA precursors by Dicer endonucleases, and function with Argonaute-family proteins to target transcript-destruction or to silence translation.

It is known that developmentally regulated molecular events following fertilization include changes in the length of the cell cycles, remodeling of chromatin structure, DNA 
methylation, synthesis of zygotic/embryonic transcripts and proteins, and degradation of maternal transcripts $[4,5]$. Various studies have shown that miRNAs regulate gene expression and also play a major role in embryo development. These RNAs have been identified in a wide range of organisms, including bovine, and plants and are conserved through species such as: C. elegans through D. melanogaster to Homo sapiens [6]. The degree to which spermatozoa miRNAs regulate fertilization and early embryonic development in bovine has yet to be discovered.

Spermatogenesis is a multistep complex developmental process. It includes continuous cell proliferation and differentiation of germ cells into final form of functional spermatozoa. This differentiation process is dependent on the sequential expression of genes. However, molecular mechanisms underlying this regulation remain largely unknown. Numerous miRNAs in germ cells are subjected to post-transcriptional and translational regulation [7]. It would be significant to identify the sperm-specific miRNA and their target genes. This will help to better understand the molecular mechanisms by which an orderly sequence of germ cell differentiation takes place by switching on and off specific genes. Knowledge on the miRNA repertoire of sperm will also expand the scope of the search for functionality of sperm-originated small RNAs in oocyte activation, fertilization and subsequent embryonic development. It is known that differences exist among bulls in their ability to fertilize and activate the egg, and for the sperm to further support embryonic development [8]. The primary focus of this study is to identify miRNAs expressed in bovine spermatozoa bulls with differing fertility potential. We used a transcripomewide strategy to compare the miRNA pools from low and high fertility bull spermatozoa and found seven differentially expressed miRNAs. Our results demonstrate that miRNAs are abundant in bovine spermatozoa. Furthermore, the levels of expression in miRNAs of high and low fertility bulls vary greatly and may be involved in controlling animal reproduction.

\section{Methods}

We analyzed the expression of miRNAs from bovine spermatozoa in a high fertility bull and a low fertility bull. This was done by isolating the total RNA from sperm cells, enrichment of miRNAs, and performing microarray analysis. The results from microarray were validated by isolating sperm RNA and performing real time RT-PCR using sperm from additional bulls with varying fertility.

\section{Semen samples and data sources}

Frozen semen samples and fertility phenotype from eight mature, progeny tested Holstein bulls with satisfactory semen quality were obtained from Alta Genetics
(Watertown, WI). The two most extreme high and low fertility bulls were used for the sperm MicroRNA MicroArray Expression Array. The other six bulls were used for the validation of the results using reverse transcriptase real time PCR.

The fertility phenotypes have been characterized in the established progeny testing program (Alta Advantage ${ }^{\circledR}$ ) which is the industry's most reliable source of fertility information. This program consisted of 180 well managed partner dairy farms with an average of 850 milking cows each located in different geographical regions across the United States. The reproductive performance of the herds are consistently monitored through accurate registration of insemination records, plus, the breeding outcomes are diagnosed by veterinary palpation or ultrasound rather than relying on non-return rates 60-90 days after breeding. Additionally, the program provides the distinct advantage of verification of paternity of the offspring using DNA testing.

\section{Bull fertility prediction}

The fertility of the sires is predicted and documented on a quarterly basis using updated results of the breeding records. For this study, the database used to predict bull fertility consisted of a total of 953,702 breeding records from 998 bulls. The breeding record for each bull was an average of 958 with a range of 300 to 12,312 . In order to rank the bulls based on their breeding values for fertility, the environmental and herd management factors that influence the fertility performance were adjusted using threshold models which were similar to previously published models by Bartel and Zwald et al. [3,9]. Parameter estimation and fertility estimations were obtained using Probit.F90 software developed by Wanget al [10]. We considered the outcome of each breeding event and adjusted the environmental factors such as the effects of herd-year-month, parity, cow, days in milk, and sire's proven status (young or proven). Then the fertility of each sire was expressed as the percent deviation of its conception rate from the average conception rates of all bulls in the database that have at least 300 breeding outcomes.

\section{Selection of high and low fertility bulls}

We used standard deviation (SD) of the predicted conception rate as the threshold values to classify a bull as high and low fertility animals [11,12]. Furthermore, the bulls selected were from the group that had highest breeding records for high reliability. Briefly, to classify high or low fertility, we used two SD values above or below the average, indicating a four SD distance range between both groups, with a minimum of 700 breeding records for each individual bull. The average fertility of high and low fertility groups were 5.2 and $-7.1 \%$ of the 
average $($ Zero $=0)$, respectively. So this allowed a $12.3 \%$ total fertility difference between high and low fertility groups. The average breeding for the high and low fertility groups were $1,419 \pm 557$ and $785 \pm 122$, respectively $($ Mean $\pm \mathrm{SD})$ (Table 1$)$.

\section{Isolation of spermatozoa}

The sperm straws were thawed together in a water bath set to $36^{\circ} \mathrm{C}$ for 30 seconds. The sperm $\left(20 \times 10^{6}\right.$ cells $)$ was poured ( 2 straws/tube) into the percoll gradient and spun at $1,509 \times g$ for 15 minutes, in ambient air at $4^{\circ} \mathrm{C}$ in the centrifuge. Motile sperm cells were isolated using percoll gradient as described previously [13]. The supernatant, containing cryoprotectants and egg yolk, was carefully removed using a micropipette. The pellet was resuspended in $1 \mathrm{ml}$ PBS and centrifuged again. The supernatant was again removed and the pellet was resuspended in $1 \mathrm{ml}$ of PBS and centrifuged at 9,500 $\mathrm{x} g$ for 1 minute at $4{ }^{\circ} \mathrm{C}$. Following this final step, the supernatant was again removed, and the sperm pellet stored at $-80^{\circ} \mathrm{C}$.

\section{Sperm RNA extraction}

RNA was isolated using Trizol (Invitrogen, Carlsbad, CA) according to Feugang et al. (2008) [12]. Briefly, $500 \mu \mathrm{l}$ of Trizol were added into each sperm cell pellet all of which were then homogenized at high speed for 30 seconds using the Pro 200 homogenizer (Pro Scientific Inc., Oxford CT). Glycogen ( $3 \mu \mathrm{l}$ of $20 \mathrm{mg} / \mathrm{ml}$ ) was added to the tubes and another $500 \mu \mathrm{l}$ of Trizol was then added followed by mixing by several pipettings. Then, the mixture was incubated for 15 minutes at $65^{\circ} \mathrm{C}$. Finally five hundred microliters of chloroform were added and is mixed again by means of several pipettings followed by vortexing for 40 seconds.

The samples were incubated at room temperature for 10 minutes followed by centrifugation for 15 minutes at $4^{\circ} \mathrm{C}$. The upper colorless phase was collected into a new sterile eppendorf tube into which five hundred microliters of $100 \%$ isopropanol were added and mixed by several pipettings.
Following incubation at $-20^{\circ} \mathrm{C}$ for an hour, the samples were vortexed for 10 seconds and incubated at room temperature for 10 minutes. The RNA was precipitated by centrifugation for 10 minutes at $4^{\circ} \mathrm{C}$. Following the removal of the supernatant, the pellet was air-dried for 5 minutes and then was rehydrated in $20 \mu \mathrm{l}$ of deionized, diethylpyrocarbonate (DEPC) treated water. The concentration of the RNA was determined using $2 \mu \mathrm{l}$ of the samples in Nanodrop ${ }^{\circledR}$ ND 1000 (NanoDrop Technologies, Wilmington, DE). The remaining $18 \mu \mathrm{l}$ of the samples were stored in $-80^{\circ} \mathrm{C}$ freezer and then shipped to Asuragen Inc. on dry ice.

\section{Enrichment of spermatozoal microRNAs and microRNA microArray expression array experiments}

Samples for miRNA profiling studies were processed by Asuragen Services [14], according to the company's standard operating procedures. Total RNA was dephosphorylated with calf intestinal phosphatase and the pCp-Biotin labeling molecule was ligated to the 3' ends of the RNA molecules. Labeled RNA was purified using BioSpin6 (Bio-Rad, Hercules CA). Hybridization, washing, staining, imaging, and signal extraction were performed according to Affymetrix-recommended procedures, except that the 20X GeneChip Eukaryotic Hybridization control cocktail was omitted from this hybridization procedure.

There were six samples processed for microarray experiments using the Ambion/Affymetrix DiscovArray. The signal processing implemented for the Ambion miRCHIP is a multi-step process involving probe specific signal detection calls, background estimate and correction, constant variance stabilization [15] and either array scaling or global normalization. For each probe, an estimated background value is subtracted that is derived from the median signal of a set of G-C-matched antigenomic controls. Arrays within a specific analysis experiment were normalized together according to the variance stabilization method described by Huber et al. (2002) [15]. Detection calls were based on a Wilcoxon rank-sum test of the miRNA probe signal compared to

Table 1 Bull information for the samples used to validate the microarray studies using real time RT-PCR

\begin{tabular}{llll}
\hline Bull no & Number of breedings & Fertility (\% difference from average fertility) & Fertility status \\
\hline $\mathbf{1}$ & 1723 & 4.4 & $\mathrm{HF}$ \\
$\mathbf{2}$ & 4,021 & 4.7 & $\mathrm{HF}$ \\
$\mathbf{3}$ & 779 & 4.4 & $\mathrm{HF}$ \\
$\mathbf{4}$ & 1,156 & 7.4 & $\mathrm{HF}$ \\
$\mathbf{5}$ & 722 & -7.5 & $\mathrm{LF}$ \\
$\mathbf{6}$ & 747 & -6.3 & $\mathrm{LF}$ \\
$\mathbf{7}$ & 704 & -5.6 & $\mathrm{LF}$ \\
$\mathbf{8}$ & 967 & -9.2 & $\mathrm{LF}$ \\
\hline
\end{tabular}


the distribution of signals from GC-content matched anti-genomic probes.

\section{Statistical analysis}

For statistical testing, a two-sample $t$-Test, with assumption of equal variance, was applied. One-way ANOVA was used for experimental designs with more than two experimental groupings or levels of the same factor. These tests define which probes are considered to be significantly differentially expressed, or "significant", based on a default p-value of 0.001 and $\log _{2}$ difference $>1$.

\section{Identification of miRNA target genes}

First, we ranked the expression levels of miRNAs for high $v s$. low fertility bulls and low vs. high fertility bulls from high to low. We selected the top 10 from the list. Then we used the miRBase database to find the related sequence for each entry by using their precursoraccession IDs [16]. Sequences were entered into the BLAT program, UCSC [17], platform and possible target genes were identified. BLAT search is designed to find sequences of $95 \%$ and greater similarity, and of length 25 bases or more. We used the human genome as a template to identify the target genes. After identification of each gene we used the HUGO Gene Nomenclature Committee (HGNC) database for annotation purposes [18].In identification of seven differentially expressed miRNAs, again we followed the same steps and presented results in Table 2.

\section{Determination of miRNA interactomes}

We used the list of predicted target genes (TOB2, CHN2, CLUL1, BC035897, BTBD2, AQP7P1) for the seven differentially expressed miRNAs as an input to Genemania [19]. Genemania is a web based tool that searches many large, publicly available biological datasets. We searched for physical interactions which cover protein-protein interactions and genetic interactions. Genemania, which uses data from BioGRID Physical, predicted protein interaction data, based on orthology from I2D and pathway and molecular interaction data from Pathway Commons. We applied default parameters and a restriction on the results applicable only to Homo sapiens.

\section{Validation of the microarray data}

Isolation of miRNAs from bull spermatozoa

Spermatozoa from eight bulls with varying fertility (Table 1) were isolated as described above. MicroRNAs were isolated from 50 million sperm cells using the mirVANA kit according to manufacturer's protocol (Ambion, Austin, TX). Briefly, sperm pellets were resuspended in the lysis/binding buffer and passed through a $25 \mathrm{G}$ needle followed by addition of Acid:Phenol: Chloroform and miRNA homogenate additive. The sample was again passed through a $25 \mathrm{G}$ needle and vortexed for $10 \mathrm{~min}$. Following $10 \mathrm{~min}$ incubation on ice, the homogenized sample was centrifuged at $10,000 \times g$ for $5 \mathrm{~min}$ at room temperature. Upper phase of the solution was carefully transferred into a new sterile tube and was mixed with $1 / 3$ ratio $(\mathrm{v} / \mathrm{v})$ of $100 \%$ ethanol. The mixture was loaded to a filter cartridge and centrifuged for $15 \mathrm{sec}$ at $10,000 \times \mathrm{g}$ at room temperature. The filtrate was collected in a fresh tube and mixed with $2 / 3$ ratio (v/v) of $100 \%$ ethanol, loaded into a new filter cartridge and centrifuged for $15 \mathrm{sec}$ at 10,000 x g. Subsequently, the filter cartridge was washed once with miRNA wash solution 1 and twice with miRNA washes solution 2. Following each washing step, the filter cartridge was centrifuged for $10 \mathrm{sec}$ at $10,000 \times \mathrm{g}$, and for one min to remove residual fluid from the filter at the last wash. Finally, the filter was transferred to a new collection tube and $50 \mu \mathrm{l}$ of elution buffer pre-heated to $95^{\circ} \mathrm{C}$ was applied to the center of the filter and centrifuged for $30 \mathrm{sec}$ at max speed to recover miRNA. Concentrations of miRNA were measured using the ND-1000 spectrophotometer (NanoDrop Technologies, Palo Alto, CA).

\section{Reverse transcriptase real time PCR}

Prior to reverse transcriptase (RT) reaction, isolated miRNAs were digested with DNAse I (1U, Promega, WI) to remove possible genomic DNA contamination. Following the digestion, 30 pg of miRNA were polyA-

Table 2 Characterization of Seven Probe Sets used for validation of microarray results using real time-RT-PCR

\begin{tabular}{llllllll}
\hline IDs & Chr & \multicolumn{1}{c}{ Sequences } & P-values & Log Ratio & Fold change D vs. R & Target Gene & \# of Physical Interactions \\
\hline hsa-aga-3155 & 22 & AAGCUUAUGGAGCAGAGGAUU & 0.00001 & 3.86 & 4.65 & TOB2 & 14 \\
hsa-aga-8197 & 7 & UGAGUGAUAAUAGGGUCGUGAC & 0.0004 & 1.22 & 5.6 & CHN2 & 2 \\
hsa-aga-6727 & 18 & UUCUGUGGCAGAUUGGGAUGGA & 0.00051 & 1.83 & 4.27 & CLUL1 & "-" \\
hsa-aga-11796 & 22 & GAGGCAGAGAAGGGACAGGAAA & 0.00073 & 1.72 & 7.35 & BC035867 & "-" \\
hsa-aga-14189 & 19 & CAUAGCGAGACCCCGUCUG & 0.00077 & 1.21 & 14.34 & BTBD2 & 3 \\
hsa-aga-6125 & 9 & UUGGAUAUUGCUUGGAGGCUCU & 0.0009 & 1.25 & 10.32 & No hits & "-" \\
hsa-aga-13659 & 9 & GACUGGAGGAGGCAUGGAGGGU & 0.0009 & 1.05 & 12.93 & AQP7P1 & "-" \\
\hline
\end{tabular}


tailed using poly (A) polymerase and then reversetranscribed using miScript Reverse Transcription Kit from Qiagen (Valencia, CA) according to the manufacturer's protocol. Briefly, the reaction was set up as following: $10 \mu \mathrm{l}$ of $2 \mathrm{X}$ QuantiTect SYBR Green PCR Master Mix, $2 \mu \mathrm{l}$ of10X miScript Universal Primer, $2 \mu \mathrm{l}$ of 10X miScript Primer Assay (Specific Primer) 1.5 pg RT product $(1 \mu \mathrm{l} \mathrm{cDNA})$ and $\mathrm{ddH}_{2} \mathrm{O}$ up to $20 \mu \mathrm{l}$ of final volume. Thermal cyclic conditions were: initial activation step at $95^{\circ} \mathrm{C}$ for $15 \mathrm{~min}$ followed by 40 cycles of denaturation, annealing and amplification $\left(94^{\circ} \mathrm{C} 15 \mathrm{sec}, 55^{\circ} \mathrm{C}\right.$ $30 \mathrm{sec}, 70^{\circ} \mathrm{C} 30 \mathrm{sec}$ ) on DNA Engine Opticon 2 Real-Time PCR Detection System (Bio-RAD, Foster City, CA). Melting curve analysis was performed as following: $95^{\circ} \mathrm{C}$ for $1 \mathrm{~min}$, then fluorescence measurement was done at every 0.8-degree increments between $60^{\circ} \mathrm{C}$ and $95^{\circ} \mathrm{C}$. In each run, a negative control with no cDNA template was included. All samples were run in duplicate for each cDNA isolation. Five different miRNAs (RNU1A, RNU6B, RNU5A, SNORD25, and SNORA73A) in the miScript PCR control set (Qiagen) were employed for normalization of miRNA expressions. The real time RTPCR experiments were repeated three times using three independent miRNAs.

\section{Statistical analyses of the real time RT-PCR}

The amounts of each isolated miRNAs for high fertility and low fertility bulls were compared by $t$-test. Data were presented as mean \pm SEM. Differences were considered significant when $\mathrm{P}$ values were lower than 0.05 . Regarding statistical analyses of the qPCR data, cycle threshold [20] values of high fertility bulls and low fertility bulls were normalized by using five reference miRNAs (RNU1A, RNU6B, RNU5A, SNORD25, SNORA73A). Normalized values for high fertility and low fertility groups were compared by using the algorithm (Relative Expression Software Tool 2009) upon which the mathematical model is based. This basis depends on the PCR efficiency of each gene investigated and the mean deviation in $\mathrm{Ct}$ between groups [21]. The expression ratios were considered statistically significant at $\mathrm{p}<0.05$.

\section{Results}

\section{MicroRNAs of bull spermatozoa}

To confirm the presence of abundant miRNAs in bull spermatozoa, the Bioanalyzer was used to determine the purity of RNA and ensure that miRNAs were indeed present in the mature sperm. For each bull there were three sample repeats and the bioanalyzer showed that small RNAs were present in each bull and their repeats (Figure 1). Small RNAs which include microRNAs were visible at $25 \mathrm{nt}$ on the gel showing an abundance of small RNAs based on the size of the band. Absence of ribosomal RNA bands suggests that the sperm RNA samples were somatic cell free.

\section{Dynamics of microRNAs in bull spermatozoa}

The miRNA samples were analyzed using a miRNA microarray (Ambion/Affymetrix DiscovArray) containing several types of probe sets derived from organisms such as plants (i.e. rock cress and soybean) and animals (i.e., zebra fish, chicken, mouse, and human). The bulls were categorized as either a high (D) or a low (R) fertility bull. Each sample is represented by a vector containing all detected probes (14215 probes). The samples showed that there were general differences between the high and low fertility bulls (Figure 2). It also, showed that there were differences among the same sample. Approximately 2510 probe sets

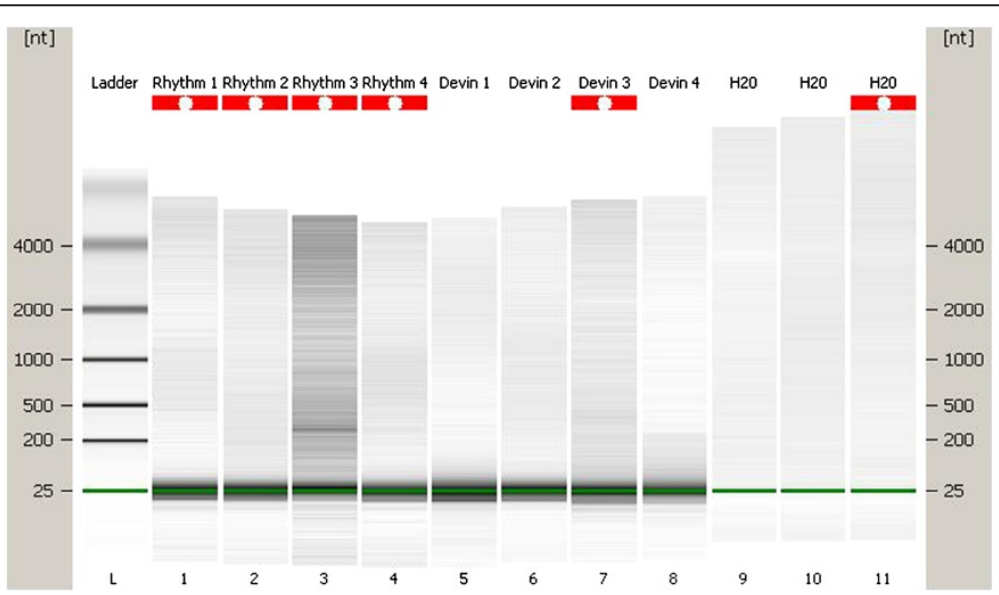

Figure 1 Small molecular weight RNA transcripts as detected using Bioanalyzer. According to the gel image there is no contamination in the RNA and the miRNA will be at or below the green line on the gel, given that miRNAs range from 17-25 nt in length. Rhythm 1, Rhythm 2, Rhythm 3, and Rhythm 4 represent biological repeats for low fertility bull of $-3.3 \pm 3.3$ fertility \%. Devin 1, Devin 2, Devin 3, and Devin 4 represent biological repeats for high fertility bull of $4 \pm 1.8$ fertility $\%$. 
were detected significantly above background (See Additional file 1: Table S1).

\section{Statistical analysis of differentially expressed microRNAs}

A heat map of detected probes is shown in Figure 2. The single horizontal red line corresponds to a nonadjusted p-value of 0.05 . Genes above this line were considered statistically significant at a p-value of 0.05 . The expression of high fertility bull (D) is compared to that of low fertility bull (R). The highly differentially expressed genes are indicated by the lines colored red. There are seven of these genes that are called significant based on an unadjusted p-value of 0.001 and a 2-fold change (Figure 2).

Each sample is represented by a vector containing only significant probes. This reduction eliminates the influence of non-significant probes to the cluster analysis. Each probe is represented in each line, with the color code confirming their differential expression levels between low and high (D) fertility bulls, despite the slight variation between replicates in each bull. Characterizations of the seven probe sets are summarized in Table 2. All the probes are from humans and their annotations are not yet completed.

\section{Identification of miRNA target genes}

In our top 10 miRNAs some probes are not human based so that we could not identify the target genes for non-human miRNAs (Tables 3 and 4). We identified several genes in the top 10 list, namely, DALRD3, C21orf34, AK091713, DNM2, and IFT80. In summary, gene ontology (GO) terms are reported for DALRD3, showing that it is related to ATP binding and nucleotide binding [22]. DNM2 has a role in biological processes such as DNA-dependent regulation of transcription, signal transduction, and/or receptor internalization. IFT80 is a protein component of intraflagellar transport (IFT) complex $\mathrm{B}$, and it is required for the formation, maintenance and functionality of cilia. We also compared our findings that are presented in Tables 3 and 4 with a recent study from Krawetz et al. [23]. In Tables 3 and 4, hsa-miR-191 was also found in the Krawetz et al. study. It was associated with genomics features; a TSS/Promoter and histones. It had been previously identified in sperm, testis, ovary, zygote and it was also reported that hsa-miR-191 was not regulated epigenetically. Another microRNA, i.e., hsa-miR-100, was also found to be associated with genomic features; TSS/Promoter. It was previously identified in testis, ovary, and zygote. It was found that hsa-miR-100 is epigenetically regulated.

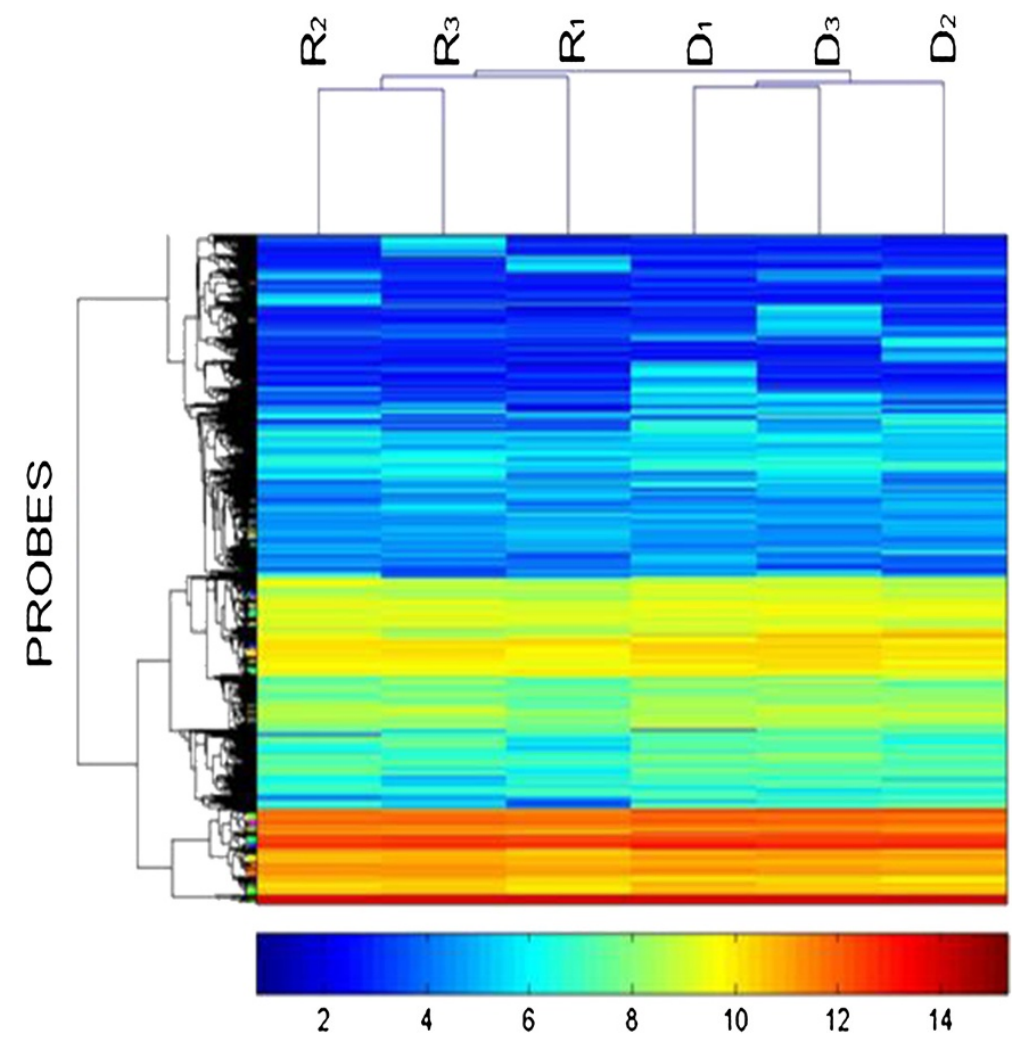

Figure 2 Heat map and the result of clustering to all MicroRNA probes detected. There are two specific groups. Column 1-3 represents bull R. Columns 4-6 represents bull D. There were differences between the two groups and some differences within the same groups. 
Table 3 Top 10 miRNAs expressed in high fertility bull (D) vs. low fertility bull (R)

\begin{tabular}{|c|c|c|c|c|c|c|c|}
\hline Probe_ID & $\begin{array}{l}\text { MicroRNA } \\
\text { Accession }\end{array}$ & $\begin{array}{l}\text { Precursor } \\
\text { Accession }\end{array}$ & D: Mean & R: Mean & D vs. R: test & $\begin{array}{l}\text { Target } \\
\text { gene }\end{array}$ & $\begin{array}{l}\text { Function of } \\
\text { target gene } \\
\text { products }\end{array}$ \\
\hline hsa-miR-191_st2 & MIMAT0000440 & MI0000465 & 13.95686623 & 13.02345008 & 0.01791063 & DALRD3 & $\begin{array}{l}\text { DALR anticodon } \\
\text { binding domain } \\
\text { containing } 3\end{array}$ \\
\hline hsa-miR-125b_st1 & MIMAT0000423 & MI0000470 & 13.91045299 & 13.02384618 & 0.01402603 & C21orf34 & \\
\hline ame-miR-125_st1 & MIMAT0001474 & MI0001578 & 13.90178917 & 12.94703119 & 0.01176129 & $\mathrm{Nl}^{*}$ & \\
\hline hsa-cand375_st1 & MIMAT0000098 & MI0000102 & 13.81001246 & 13.37153817 & 0.13644647 & AK091713 & \\
\hline hsa-miR-638_st2 & MIMAT0003308 & MI0003653 & 13.78857283 & 13.96774448 & 0.0187295 & DNM2 & dynamin 2 \\
\hline hsa-miR-100_st2 & MIMAT0000098 & MI0000102 & 13.59036572 & 13.0028788 & 0.07920135 & AK091713 & \\
\hline dps-miR-289_st1 & MIMAT0001253 & MI0001348 & 13.21245856 & 13.84341616 & 0.0205619 & $\mathrm{Nl}^{*}$ & \\
\hline hsa-miR-15b_st1 & MIMAT0000417 & MI0000438 & 13.19413812 & 11.97871936 & 0.07635284 & IFT80 & $\begin{array}{l}\text { intraflagellar } \\
\text { transport } 80 \\
\text { homolog }\end{array}$ \\
\hline dme-miR-100_st1 & MIMAT0000357 & MI0000378 & 12.89301651 & 12.12274822 & 0.05917641 & $\mathrm{Nl}^{*}$ & \\
\hline dre-miR-15b_st1 & MIMAT0001773 & MI0001893 & 12.66899194 & 11.24401476 & 0.04350151 & $\mathrm{NI}^{*}$ & \\
\hline
\end{tabular}

Among the seven differentially expressed miRNAs from the array we were able to verify six of them and these are TOB2, CHN2, CLUL1, BC035897, BTBD2, and AQP7P1. The first, TOB2 belongs to family of antiproliferative proteins, which are involved in the regulation of cell cycle progression [24]. Like the TOB protein, TOB2 inhibits cell cycle progression from the G0/G1 to $S$ phases. CHN2 has GTPase-activating protein activity that plays a significant role in the proliferation and migration of smooth muscle cells. CLUL1 is related with cell death. BTBD2 protein binds Topoisomerase I. In addition, it is stated in the literature that polymorphisms of LIG4, BTBD2, HMGA2, and RTEL1 genes are involved in the double-strand break repair pathway (PMID 20368557).

\section{Determination of miRNA interactomes}

Gene networks that are composed of miRNAs and their target mRNAs may represent important possible biological functions. In our analysis we found interactions for 3 genes from the list above, they are TOB1, CHN2 and BTBD2. In addition, TOB2 has 12 possible proteinprotein interactions. Two of these interactions represent stronger associations than the rest and these two most significant interactions occur between TOB2-CNOT7 and TOB2-RPS6KA1 as shown in Figure 3A. TOB2 is a transducer of ERBB2 and belongs to the TOB/BTG1 family of antiproliferative proteins that are involved in the regulation of cell cycle progression. Another significant finding about CHN2 is shown in Figure 3B. This gene has interactions with EPHA1 and EFNA2. Both

Table 4 Top 10 miRNAs expressed in low fertility bull (R) vs. high fertility bull (D)

\begin{tabular}{|c|c|c|c|c|c|c|c|}
\hline Probe_ID & $\begin{array}{l}\text { MicroRNA } \\
\text { Accession }\end{array}$ & $\begin{array}{l}\text { Precursor } \\
\text { Accession }\end{array}$ & D: Mean & R: Mean & D vs. R: ttest & Target Gene & $\begin{array}{c}\text { Function of } \\
\text { target gene } \\
\text { products }\end{array}$ \\
\hline hsa-miR-638_st2 & MIMAT0003308 & MI0003653 & 13.78857283 & 13.96774448 & 0.0187295 & DNM2 & dynamin 2 \\
\hline dps-miR-289_st1 & MIMAT0001253 & MI0001348 & 13.21245856 & 13.84341616 & 0.0205619 & $\mathrm{Nl}^{*}$ & \\
\hline hsa-cand375_st1 & MIMAT0000098 & Ml0000102 & 13.81001246 & 13.37153817 & 0.13644647 & AK091713 & \\
\hline hsa-miR-125b_st1 & MIMAT0000423 & MI0000470 & 13.91045299 & 13.02384618 & 0.01402603 & $\mathrm{~N} l^{*}$ & \\
\hline hsa-miR-191_st2 & MIMAT0000440 & Ml0000465 & 13.95686623 & 13.02345008 & 0.01791063 & C21orf34 & \\
\hline hsa-miR-100_st2 & MIMAT0000098 & MI0000102 & 13.59036572 & 13.0028788 & 0.07920135 & AK091713 & \\
\hline hcmv-miR-UL70-3p_st2 & MIMAT0003343 & Ml0003688 & 12.62649632 & 12.99272922 & 0.00473263 & $N l^{*}$ & \\
\hline ame-miR-125_st1 & MIMAT0001474 & MI0001578 & 13.90178917 & 12.94703119 & 0.01176129 & $\mathrm{Nl}^{*}$ & \\
\hline dme-miR-100_st1 & MIMAT0000357 & Ml0000378 & 12.89301651 & 12.12274822 & 0.05917641 & $\mathrm{~N}^{*}$ & \\
\hline hsa-miR-15b_st1 & MIMAT0000417 & MI0000438 & 13.19413812 & 11.97871936 & 0.07635284 & IFT80 & $\begin{array}{l}\text { intraflagellar } \\
\text { transport } \\
80 \text { homolog }\end{array}$ \\
\hline
\end{tabular}




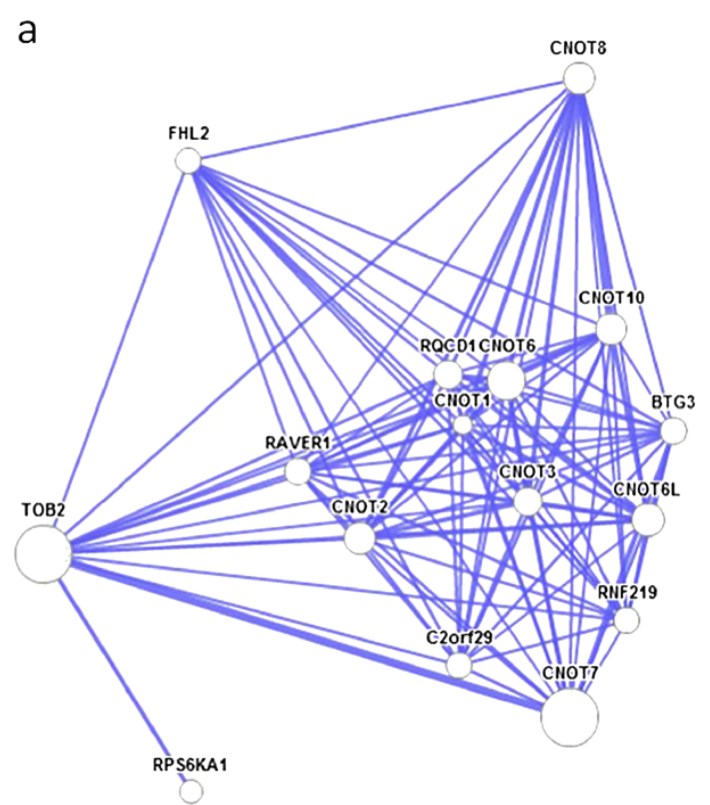

b

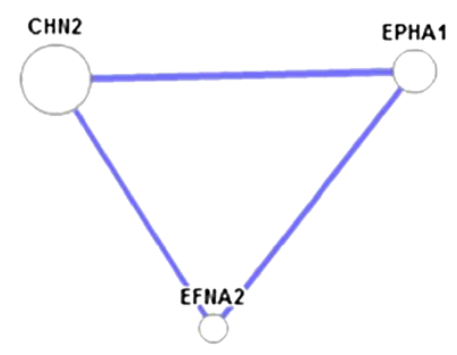

C

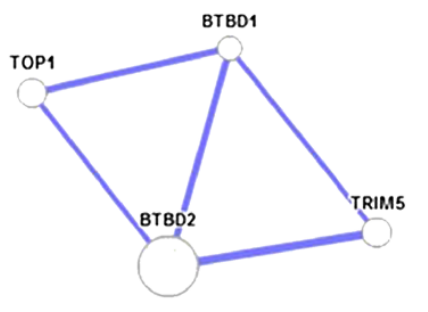

Figure 3 Interactome of miRNA targets. Figures present predicted physical (protein-protein) interactions of a) TOB2 b) CHN2 c) BTBD2.

EPHA1 and EFNA2 have been implicated in mediating developmental events, particularly in the nervous system. BTBD2 has physical interactions between TOP1, BTBD1and TRIM5 as indicated in Figure 3C.

\section{Validation of the microarray data using real time RT-PCR}

Total amounts of miRNAs isolated from 50 million sperm cells from the six bulls ranged between $148.6 \pm 9.6$ and $173.3 \pm 26.3$ which shows there were not significant differences among the bulls. Optic density of 260/280 in the Nanodrop measurement was $1.7 \pm 0.1$ for the isolated miRNAs.

Real time RT-PCR analyses confirmed that miRNA expressions of hsa-asg-14189 (3.6 times), hsa-asg-8197 (4.5 times), hsa-asg-6125 (3.6 times), hsa-asg-6727 (3.9 times), and, hsa-asg-11796 (3.5 times) shows they were more abundant in the low fertility group when compared to the high fertility group ( $\mathrm{p}<0.0001$, Figure 4$)$. In contrast, the transcript levels of hsa-asg-3155 and hsaasg-13659 did not show any difference between low fertility and high fertility groups by Real time RT-PCR.

\section{Discussion}

MicroRNAs play an essential part of animal gene regulatory networks and a given genome could encode nearly 1000 miRNAs $[20,26]$. These short transcripts provide functions, in animals, that are essential for normal development and cellular homeostasis [27]. In this study, we identified miRNAs present in bovine spermatozoa. While examining two groups of bulls having different fertility we found that there was an abundance of
miRNAs in bovine spermatozoa. However, only a few (seven) miRNAs were significantly differentially expressed. These seven miRNAs had a probe set which were from Homo sapiens. This could possibly mean that the sequences are conserved through species of Bos taurus and Homo sapiens although some miRNAs appear to be species specific [20]. However, among the seven significantly expressed miRNAs that were identified, there were none with published annotations; therefore, at the present time, their functions are unknown.

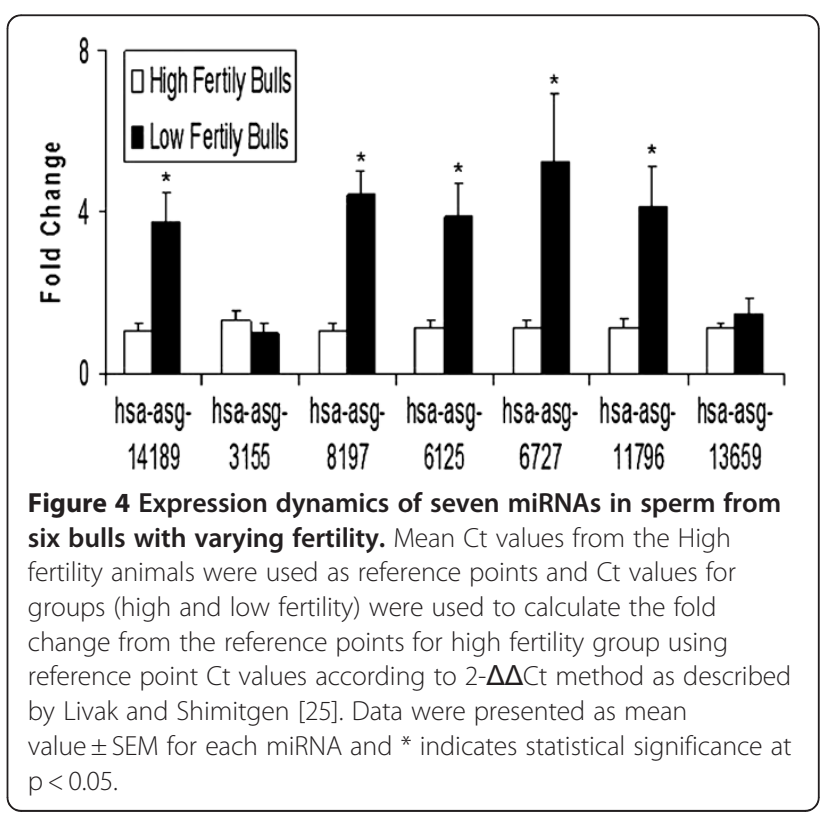


Nevertheless, some studies have given explanations for possible functions of miRNAs through overexpression, misexpression, and in vitro knockdown [28].

In our studies, we observed that miRNAs detected in low fertility bull were expressed at higher levels than those in the high fertility bull. The higher expression levels of the miRNA in the low fertility bulls could mean that miRNAs might be down regulating expression of genes whose products play important roles in fertilization and early embryonic development. For example, recently it has been demonstrated that sperm borne miRNAs have been transferred into the oocyte and that miR34c is involved in the first cleavage division in mouse [29]. In this study we found that there were some variations of miRNA expressions within the same groups of bulls. This could possibly be due to the collection times, other variables such as nutrition or animal health, or technical variations. Semen was collected within the three month period and shipped to our laboratory. It is possible that gene-environment interactions among other factors might contribute to differences in gene expression including miRNAs in different ejaculates. Another limitation was that the miRNA microarray contained probes for known miRNAs for humans, mice and rats. Thus, it is expected that additional and bovine specific miRNAs would have been detected using bovine specific miRNA microarrays if they had been available. In spermatogenesis, there are many miRNAs that are waiting to be detected but little is known about their expression level or patterns [30].

Molecular interactions contribute to the homeostasis and the performance of a cell. Networking between genes, RNA, proteins and other biological molecules represent the interactome of a cell. Interactomic approach sheds light on miRNA as a regulator of networking proteins and will give the functional annotations of the molecules involved. Because of the interconnectivity among other proteins and the expression of miRNA (hsa-aga-6125) against BTBD2, it is possible that this miRNA might be the master regulator for expression of this set of genes. Co-expression of the miRNAs might be regulating the physical interactions among the networks of genes such as CHN2-EPHA1EFNA2 (Tables 3 and 4). The target gene, AQP7P1 of probe ID hsa-aga-13659 is a member of the Aquaporin gene family. It encodes for Aquaporin 7 pseudogene 1, an integral membrane protein playing a role as a channel for aqueous flow across the membrane. Aquaporin 7 (AQP7) is known to be expressed in human sperm tails and lack of its expression has also been observed in some infertile patients. AQP7 positive sperm have significantly higher motility rate than that of AQP7 negative sperm, making this gene expression one of the deciding factors for male infertility [31]. IFT80 gene expression is determined to be targeted by hsamiR-15b_st1. This gene product is a component of the intraflagellar transport complex which is essential for the development of motile cilia [32]. Probe ID involved in Dynamin 2 (DNM2) protein regulation is hsa-miR-638_st2. Dynamin 2 is found in the acrosomal region of the sperm, indicating its possible role during fertilization [33].

To what extend sperm miRNAs target sperm mRNAs? For this quest, we looked for targets of the top 10 miRNAs in the sperm mRNA data reported in the literature. In this present study we are reporting the genes that are the predicted targets of miRNAs which are listed in the Tables 3and 4. We searched target sperm mRNAs in the current literature for our target genes that were led by the top 10 miRNAs and we did not find any matches $[2,34,35]$. Therefore, the genes that we are reporting in this paper may have been potential targets for an extended study for a sperm mRNA focused study.

It is clear that miRNAs are abundant in bovine spermatozoa. Determining the function(s) of the significantly differentially expressed miRNAs will help us understand functional genomics of bovine spermatozoa. Distinct bovine miRNAs have been identified in bovine adipose tissue that should be useful for studying the role of miRNAs in cattle and for comparative genomic analysis of miRNA function and regulations [17]. Identifying specific miRNAs expressed in spermatozoa of different fertility, will help to better understand mammalian gametogenesis. From this we will be able to develop a model which will aid in understanding the roles of miRNAs during early development both in other animals, as well as, mammals including humans and bovine studied here. It would be interesting to identify target mRNAs of these miRNAs detected in bull spermatozoa. This study provides the groundwork for uncovering the role of miRNA in differential fertility. Future studies will include target prediction for the identified spermatozoal miRNA and thus develop a miRNA based molecular biomarker for fertility.

\section{Conclusions}

In addition to providing half of the genomic DNA, research shows that the male gamete harbors large amounts of miRNAs. These small transcripts may have roles in sperm physiology including fertilization, egg activation and embryo development.

\section{Additional file}

Additional file 1: Table S1. Complete microarray output for the

samples.

Competing interests

Authors declare that they have no competing interests in this study. 


\section{Authors' contributions}

EM designed the study; AG did bioinformatics analyses and helped write the paper; LR helped write the paper; OA performed the real time RT-PCR, AK and ET provided sperm from high and low fertility bulls, provided the bull fertility data, and helped write the paper; EAC helped with data analyses, bioinformatics and manuscript writing; AU, JP and AP performed bioinformatics analyses and helped write the paper. All authors read and approved the final manuscript.

\section{Acknowledgements}

This study was funded in part by Mississippi Agriculture, Forestry, and Experiment Station, and by Alta Genetics, Inc. This work was supported in part by the National Science Foundation under grant DBI-1004842. AP was supported by the National Science foundation under grant EPS-0903787 and by the Mississippi INBRE funded by grants from the National Center for Research Resources (5P2ORR016476-11) and the National Institute of General Medical Sciences (8 P20 GM103476-11) from the National Institutes of Health.

\section{Author details}

${ }^{1}$ Departments of Animal and Dairy Sciences, Mississippi State University, Mississippi State, MS 39762, USA. 'Brown University, Providence, RI 02912, USA. ${ }^{3}$ University of Wisconsin Madison, Madison, WI 53706, USA.

${ }^{4}$ Department of Obstetrics and Gynecology, Faculty of Veterinary Medicine, Dicle University, Diyarbakir, Turkey. ${ }^{5}$ Alta Genetics, Inc, Watertown, WI 53094 USA. ${ }^{6} \mathrm{New}$ College of Florida, Sarasota, FL, USA. ${ }^{7}$ Computer Sciences and Engineering, Mississippi State University, Mississippi State, MS 39762, USA.

\section{Received: 11 April 2012 Accepted: 29 August 2012}

Published: 14 September 2012

\section{References}

1. Dejarnette JM: The effect of semen quality on reproductive efficiency. Vet Clin North Am Food Anim Pract 2005, 21:409-418.

2. Feugang JM, Rodriguez-Osorio N, Kaya A, Wang H, Page G, Ostermeier GC, Topper EK, Memili E: Transcriptome analysis of bull spermatozoa: implications for male fertility. Reprod Biomed Online 2010, 21:312-324.

3. Bartel DP: MicroRNAs: target recognition and regulatory functions. Cell 2009, 136:215-233.

4. Memili E, First NL: Control of gene expression at the onset of bovine embryonic development. Biol Reprod 1999, 61:1198-1207.

5. Misirlioglu M, Page GP, Sagirkaya H, Kaya A, Parrish JJ, First NL, Memili E: Dynamics of global transcriptome in bovine matured oocytes and preimplantation embryos. Proc Natl Acad Sci U S A 2006, 103:18905-18910.

6. Baskerville S, Bartel DP: Microarray profiling of microRNAs reveals frequent coexpression with neighboring miRNAs and host genes. RNA 2005, 11:241-247.

7. Bouhallier F, Allioli N, Lavial F, Chalmel F, Perrard MH, Durand P, Samarut J, Pain B, Rouault JP: Role of miR-34c microRNA in the late steps of spermatogenesis. RNA 2010, 16:720-731.

8. Saacke RG: Sperm morphology: Its relevance to compensable and uncompensable traits in semen. Theriogenology 2008, 70:473-478.

9. Zwald NR, Weigel KA, Chang YM, Welper RD, Clay JS: Genetic selection for health traits using producer-recorded data. II. Genetic correlations, disease probabilities, and relationships with existing traits. J Dairy Sci 2004, 87:4295-4302.

10. Wang C, Yang C, Chen X, Yao B, Zhu C, Li L, Wang J, Li X, Shao Y, Liu Y, et al: Altered profile of seminal plasma microRNAs in the molecular diagnosis of male infertility. Clin Chem 2011, 57:1722-1731.

11. Peddinti D, Nanduri B, Kaya A, Feugang JM, Burgess SC, Memili E: Comprehensive proteomic analysis of bovine spermatozoa of varying fertility rates and identification of biomarkers associated with fertility. BMC Syst Biol 2008, 2:19.

12. Feugang JM, Camargo-Rodríguez O, Memili E: Culture systems for bovine embryos. Livest Sci 2009, 121:141-149.

13. Sagirkaya H, Misirlioglu M, Kaya A, First NL, Parrish JJ, Memili E: Developmental and molecular correlates of bovine preimplantation embryos. Reproduction 2006, 131:895-904

14. Asuragen. Austin, TX: http://www.asuragen.com.

15. Huber W, von Heydebreck A, Sultmann H, Poustka A, Vingron M: Variance stabilization applied to microarray data calibration and to the quantification of differential expression. Bioinformatics 2002, 18(Suppl 1): S96-S104.

16. Rusinov V, Baev V, Minkov IN, Tabler M: Microlnspector: a web tool for detection of miRNA binding sites in an RNA sequence. Nucleic Acids Res 2005, 33:W696-W700.

17. Gu Z, Eleswarapu S, Jiang H: Identification and characterization of microRNAs from the bovine adipose tissue and mammary gland. FEBS Lett 2007, 581:981-988.

18. Seal RL, Gordon SM, Lush MJ, Wright MW, Bruford EA: Genenames.org: the HGNC resources in 2011. Nucleic Acids Res 2011, 39:D514-D519.

19. Strozzi F, Mazza R, Malinverni R, Williams JL: Annotation of 390 bovine miRNA genes by sequence similarity with other species. Anim Genet 2009, 40:125.

20. Bentwich I, Avniel A, Karov Y, Aharonov R, Gilad S, Barad O, Barzilai A, Einat $P$, Einav $U$, Meiri E, et al: Identification of hundreds of conserved and nonconserved human microRNAs. Nat Genet 2005, 37:766-770.

21. Pfaffl MW, Horgan GW, Dempfle L: Relative expression software tool (REST) for group-wise comparison and statistical analysis of relative expression results in real-time PCR. Nucleic Acids Res 2002, 30:e36.

22. Ashburner M, Ball CA, Blake JA, Botstein D, Butler H, Cherry JM, Davis AP, Dolinski K, Dwight SS, Eppig JT, et al: Gene ontology: tool for the unification of biology. The Gene Ontology Consortium. Nat Genet 2000, 25:25-29.

23. Krawetz SA, Kruger A, Lalancette C, Tagett R, Anton E, Draghici S, Diamond MP: A survey of small RNAs in human sperm. Hum Reprod 2011, 26:3401-3412.

24. Ikematsu N, Yoshida Y, Kawamura-Tsuzuku J, Ohsugi M, Onda M, Hirai M, Fujimoto J, Yamamoto T: Tob2, a novel anti-proliferative Tob/BTG1 family member, associates with a component of the CCR4 transcriptional regulatory complex capable of binding cyclin-dependent kinases. Oncogene 1999, 18:7432-7441.

25. Livak KJ, Schmittgen TD: Analysis of relative gene expression data using real-time quantitative PCR and the 2(-Delta Delta C $(T))$ Method. Methods 2001, 25:402-408.

26. Bartel DP: MicroRNAs: genomics, biogenesis, mechanism, and function. Cell 2004, 116:281-297.

27. Hwang HW, Wentzel EA, Mendell JT: Cell-cell contact globally activates microRNA biogenesis. Proc Natl Acad Sci U S A 2009, 106:7016-7021.

28. Harfe BD: MicroRNAs in vertebrate development. Curr Opin Genet Dev 2005, 15:410-415.

29. Liu WM, Pang RT, Chiu PC, Wong BP, Lao K, Lee KF, Yeung WS: Sperm-borne microRNA-34c is required for the first cleavage division in mouse. Proc Natl Acad Sci U S A 2012, 109:490-494.

30. Yan N, Lu Y, Sun H, Tao D, Zhang S, Liu W, Ma Y: A microarray for microRNA profiling in mouse testis tissues. Reproduction 2007, 134:73-79.

31. Saito K, Kageyama Y, Okada Y, Kawakami S, Kihara K, Ishibashi K, Sasaki S: Localization of aquaporin-7 in human testis and ejaculated sperm: possible involvement in maintenance of sperm quality. J Urol 2004, 172:2073-2076.

32. Rix S, Calmont A, Scambler PJ, Beales PL: An Ift80 mouse model of short rib polydactyly syndromes shows defects in hedgehog signalling without loss or malformation of cilia. Hum Mol Genet 2011, 20:1306-1314

33. Zhao L, Shi X, Li L, Miller DJ: Dynamin 2 associates with complexins and is found in the acrosomal region of mammalian sperm. Mol Reprod Dev 2007, 74:750-757.

34. Lalancette C, Thibault C, Bachand I, Caron N, Bissonnette N: Transcriptome analysis of bull semen with extreme nonreturn rate: use of suppressionsubtractive hybridization to identify functional markers for fertility. Biol Reprod 2008, 78:618-635.

35. Bissonnette N, Levesque-Sergerie JP, Thibault C, Boissonneault G: Spermatozoal transcriptome profiling for bull sperm motility: a potential tool to evaluate semen quality. Reproduction 2009, 138:65-80.

doi:10.1186/1477-7827-10-82

Cite this article as: Govindaraju et al:: Dynamics of microRNAs in bull spermatozoa. Reproductive Biology and Endocrinology 2012 10:82. 